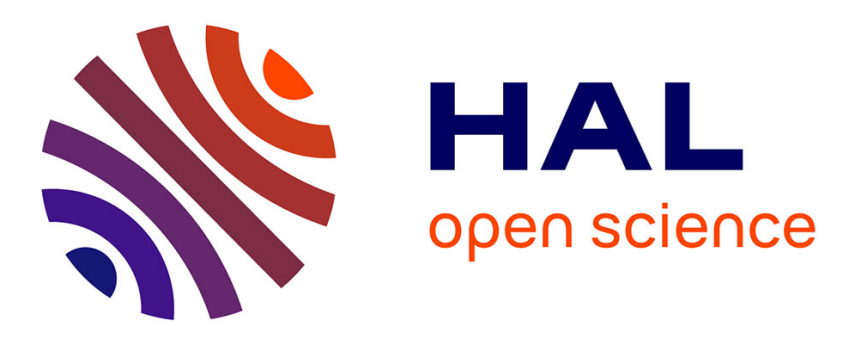

\title{
A finite volume scheme for a seawater intrusion model with cross-diffusion
}

\author{
Ahmed Ait Hammou Oulhaj
}

\section{To cite this version:}

Ahmed Ait Hammou Oulhaj. A finite volume scheme for a seawater intrusion model with crossdiffusion. FVCA8 2017 - International Conference on Finite Volumes for Complex Applications 8, Jun 2017, Lille, France. pp.421-429, 10.1007/978-3-319-57397-7_35 . hal-01541229

\section{HAL Id: hal-01541229 \\ https://hal.science/hal-01541229}

Submitted on 18 Jun 2017

HAL is a multi-disciplinary open access archive for the deposit and dissemination of scientific research documents, whether they are published or not. The documents may come from teaching and research institutions in France or abroad, or from public or private research centers.
L'archive ouverte pluridisciplinaire HAL, est destinée au dépôt et à la diffusion de documents scientifiques de niveau recherche, publiés ou non, émanant des établissements d'enseignement et de recherche français ou étrangers, des laboratoires publics ou privés. 


\title{
A finite volume scheme for a seawater intrusion model with cross-diffusion
}

\author{
Ahmed Ait Hammou Oulhaj
}

\begin{abstract}
We consider a finite volume scheme for a seawater intrusion model. It is based on a two-point flux approximation with upwind mobilities. The scheme preserves at the discrete level the main features of the continuous problem: the nonnegativity of the solutions, the decay of the energy and the control of the entropy and its dissipation. Moreover the scheme converges towards a weak solution to the problem. Numerical results are provided to illustrate the behavior of the model and of the scheme.
\end{abstract}

Key words: Finite Volume scheme, entropy stability, decay of energy.

MSC (2010): 65M08, 65N08, 35Q30

\section{The continuous problem and objectives}

Let $\Omega$ be a polygonal open bounded and connected subset of $\mathbb{R}^{2}$, and $T>0$ be a finite time horizon. We consider the following cross-diffusion system of degenerate parabolic equations

$$
\begin{cases}\partial_{t} f-\nabla \cdot(\mu f \nabla(f+g+b))=0 & \text { in } \quad \Omega_{T}:=\Omega \times(0, T), \\ \partial_{t} g-\nabla \cdot(g \nabla(\mu f+g+b))=0 & \text { in } \quad \Omega_{T}, \\ \nabla f \cdot \mathbf{n}=\nabla g \cdot \mathbf{n}=0, & \text { on } \partial \Omega \times(0, T), \\ f_{\mid t=0}=f_{0} \geq 0, \quad g_{\mid t=0}=g_{0} \geq 0, & \text { in } \Omega .\end{cases}
$$

Ahmed Ait Hammou Oulhaj

Laboratoire Paul Painlevé, UMR CNRS 8524

Université Lille 1, 59655 Villeneuve d'Ascq Cedex

e-mail: ahmed.ait-hammou-oulhaj@math.univ-lille1.fr 
It models the seawater intrusion in an unconfined aquifer (see [4]). We assume that the impermeable interface between the saltwater and the bedrock is given by $z=b(x)$. The saltwater and the freshwater are assumed to be immiscible. The interfaces between the salt-and freshwater, and between the freshwater and the dry soil is located at height $\mathrm{z}=\mathrm{g}(\mathrm{x}, \mathrm{t})+\mathrm{b}(\mathrm{x})$ and $\mathrm{z}=\mathrm{f}(\mathrm{x}, \mathrm{t})+\mathrm{g}(\mathrm{x}, \mathrm{t})+\mathrm{b}(\mathrm{x})$ respectively (cf. Fig. $1)$. The parameter $\mu$ is given by $\mu=\frac{\rho_{\text {fresh }}}{\rho_{\text {salt }}} \in(0,1)$, with $\rho$ the mass density of the fluid. The initial data $f_{0}$ and $g_{0}$ are assumed to belong to $L^{\infty}(\Omega)$.

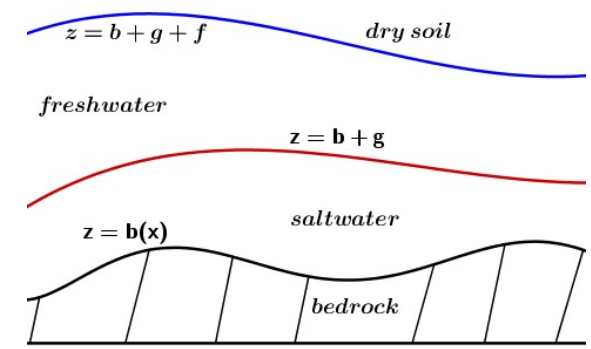

Fig. 1 Description of an unconfined aquifer

Following [2, 6, 5], there exists a weak solution to the problem (1). Moreover

$$
f \geq 0, g \geq 0 \text { a.e in } \Omega_{T} .
$$

We recall the definition of entropy (resp. energy) functional introduced in $[2,6]$ :

$$
\begin{gathered}
\mathfrak{H}(f, g)=\int_{\Omega}\left[\Gamma(g)+\frac{1}{\mu} \Gamma(f)\right] \mathrm{d} x, \quad \text { where } \Gamma(s)=s \log s-s+1, \\
\mathfrak{E}(f, g)=\int_{\Omega}\left[\frac{\mu}{2}(f+g+b)^{2}+\frac{1-\mu}{2}(g+b)^{2}\right] \mathrm{d} x .
\end{gathered}
$$

Multiplying (formally) the first equation of (1) by $\frac{1}{\mu} \log f($ resp. $\mu(f+g+b))$ and the second equation by $\log g$ (resp. $\mu f+g+b$ ), integrating over $\Omega$ and summing both equalities, get the classical entropy/dissipation property:

$$
\frac{\mathrm{d}}{\mathrm{d} t} \mathfrak{H}(f, g)+\frac{1-\mu}{2} \int_{\Omega}\left[(\nabla f)^{2}+(\nabla g)^{2}\right] \mathrm{d} x \leq \frac{1}{2(\mu+1)} \int_{\Omega}(\nabla b)^{2} \mathrm{~d} x,
$$

and the decay of the energy functional along time:

$$
\frac{\mathrm{d}}{\mathrm{d} t} \mathfrak{E}(f, g)+\int_{\Omega}\left[\mu^{2} f(\nabla(f+g+b))^{2}+g(\nabla(\mu f+g+b))^{2}\right] \mathrm{d} x=0 .
$$


In this work, We propose a finite volume scheme for the problem (1). This scheme is based on a two-point flux approximation with upwind mobilities. It is designed in order to preserve at the discrete level the main features of the continuous problem: the nonnegativity of the solutions (2), the decay of the energy (4), and the control of the entropy and of its dissipation (3). Based on these estimates, the convergence of the scheme can be proved. We refer to [1] for this purpose.

\section{The finite volume scheme}

An admissible mesh of $\Omega$ is given by a family $\mathscr{T}$ of a control volumes (open and convex polygons), a family $\mathscr{E}$ of edges, and a family of points $\left(x_{K}\right)_{K \in \mathscr{T}}$ which satisfy Definition 9.1 in [3]. This definition implies that the straight line between two neighboring centers of cells $\left(x_{K}, x_{L}\right)$ is orthogonal to the edge $\sigma=K \mid L$.

We distinguish the interior edges $\sigma \in \mathscr{E}_{\text {int }}$ and the boundary edges $\sigma \in \mathscr{E}_{\text {ext }}$. The set of edges $\mathscr{E}$ equals the union $\mathscr{E}$ int $\cup \mathscr{E}_{\text {ext }}$. For a control volume $K \in \mathscr{E}$, we denote by $\mathscr{E}_{K}$ the set of its edges, by $\mathscr{E}_{\text {int }, K}$ the set of its interior edges, and by $\mathscr{E}_{\text {ext }, K}$ the set of edges of $K$ included in $\partial \Omega$.

Furthermore, we denote by $d$ the distance in $\mathbb{R}^{2}$ and by $m$ the Lebesgue measure in $\mathbb{R}^{2}$ or $\mathbb{R}$. We assume that the mesh satisfies the following regularity requirement: there exists $\zeta>0$ such that it holds

$$
d\left(x_{K}, \sigma\right) \geq \zeta d\left(x_{K}, x_{L}\right), \quad \forall K \in \mathscr{T}, \forall \sigma \in \mathscr{E}_{\text {int }, K} \text {, with } \sigma=K \mid L .
$$

For all $\sigma \in \mathscr{E}_{\text {int, } K}$, with $\sigma=K \mid L$, we define $d_{\sigma}=d\left(x_{K}, x_{L}\right)$, and the transmissibility coefficient $\tau_{\sigma}=\frac{m(\sigma)}{d_{\sigma}}, \quad \sigma \in \mathscr{E}$. The size of the mesh is $\delta=\max _{K \in \mathscr{T}}(\operatorname{diam}(K))$. Let $N$ be a positive integer, and $\Delta t=\frac{T}{N}$; then a uniform discretization of $(0, T)$ is given by the family $\left(t^{n}\right)_{n \in\{0, \ldots, N\}}$ where $t^{n}=n \Delta t$.

We denote by $\mathscr{D}$ an admissible space-time discretization of $\Omega_{T}$ composed of an admissible mesh $\mathscr{T}$ of $\Omega$ and the values $\Delta t$ and $N$. The size of this space-time discretization $\mathscr{D}$ is defined by $\eta=\max (\delta, \Delta t)$.

Remark 1. Voronoï meshes and triangular meshes with uniformly acute angles are a typical examples of admissible meshes satisfying (5).

The initial conditions are discretized by

$$
s_{\mathscr{T}}^{0}=\sum_{K \in \mathscr{T}} s_{K}^{0} \mathbf{1}_{K}, \quad \text { where } s_{K}^{0}=\frac{1}{m(K)} \int_{K} s_{0}(x) \mathrm{d} x, \quad \forall K \in \mathscr{T}, \quad \text { with } s=f \text { or } g,
$$

and $\mathbf{1}_{K}$ is the characteristic function on $K$. The discretization of problem (1) is given by the following set of nonlinear equations: for $K \in \mathscr{T}$ and $0 \leq n \leq N-1$ 


$$
\begin{gathered}
m(K) \frac{f_{K}^{n+1}-f_{K}^{n}}{\Delta t}+\sum_{\sigma \in \mathscr{E}_{\text {int }, K}} \tau_{\sigma} f_{\sigma}^{n+1} \mu\left(u_{K}^{n+1}-u_{L}^{n+1}\right)=0 \\
m(K) \frac{g_{K}^{n+1}-g_{K}^{n}}{\Delta t}+\sum_{\sigma \in \mathscr{E}_{\text {int }, K}} \tau_{\sigma} g_{\sigma}^{n+1}\left(v_{K}^{n+1}-v_{L}^{n+1}\right)=0
\end{gathered}
$$

where $u_{K}^{n+1}=f_{K}^{n+1}+g_{K}^{n+1}+b_{K}, v_{K}^{n+1}=\mu f_{K}^{n+1}+g_{K}^{n+1}+b_{K}$, with $b_{K}=b\left(x_{K}\right)$,

$$
f_{\sigma}^{n+1}=\left\{\begin{array}{ll}
\left(f_{K}^{n+1}\right)^{+} & \text {if } u_{K}^{n+1} \geq u_{L}^{n+1}, \\
\left(f_{L}^{n+1}\right)^{+} & \text {if } u_{K}^{n+1}<u_{L}^{n+1},
\end{array} \quad g_{\sigma}^{n+1}= \begin{cases}\left(g_{K}^{n+1}\right)^{+} & \text {if } v_{K}^{n+1} \geq v_{L}^{n+1} \\
\left(g_{L}^{n+1}\right)^{+} & \text {if } v_{K}^{n+1}<v_{L}^{n+1}\end{cases}\right.
$$

with $x^{+}=\max (x, 0)$.

Proposition 1. (existence of a discrete solution) For $n \in\{0, \ldots, N\}$ there exists (at least) one solution of the scheme (6)-(8). Moreover $f_{K}^{n} \geq 0, g_{K}^{n} \geq 0$, for $K \in \mathscr{T}$.

Proof. Let us to prove the nonnegativity of $f_{K}^{n}$ (which is similar for $g_{K}^{n}$ ). This property clearly holds for $n=0$. Assume now the nonnegativity holds at time step $n$, and assume that $f_{K}^{n+1}<0$, for some $K \in \mathscr{T}$. In view of the definition (8) of $f_{\sigma}^{n+1}$ one has that

$f_{K}^{n+1}=f_{K}^{n}-\frac{\mu \Delta t}{m(K)} \sum_{\sigma \in \mathscr{E}_{\mathrm{int}, K}} \tau_{\sigma} \underbrace{\left(f_{K}^{n+1}\right)^{+}}_{=0}\left(u_{K}^{n+1}-u_{L}^{n+1}\right)^{+}-\left(f_{L}^{n+1}\right)^{+}\left(u_{K}^{n+1}-u_{L}^{n+1}\right)^{-}) \geq 0$,

yielding a contradiction, ensuring that $f_{K}^{n+1} \geq 0, \quad \forall K \in \mathscr{T}, \forall n \geq 0$. The proof of existence is detailed in [1].

\section{Entropy and energy estimates}

We introduce a discrete version of entropy (resp. energy) functional:

$$
\begin{gathered}
\mathfrak{H}^{n}:=\mathfrak{H}\left(f_{K}^{n}, g_{K}^{n}\right)=\sum_{K \in \mathscr{T}} m(K)\left(\frac{1}{\mu} \Gamma\left(f_{K}^{n}\right)+\Gamma\left(g_{K}^{n}\right)\right), \\
\mathfrak{E}^{n}:=\mathfrak{E}\left(f_{K}^{n}, g_{K}^{n}\right)=\sum_{K \in \mathscr{T}} m(K)\left(\frac{\mu}{2}\left(f_{K}^{n}+g_{K}^{n}+b_{K}\right)^{2}+\frac{1-\mu}{2}\left(g_{K}^{n}+b_{K}\right)^{2}\right) .
\end{gathered}
$$

We etasblish now the discrete countrepart of (3) and (4).

Proposition 2. There exists $C$ depending only on $T, \Omega$ and $b$ such that

$$
\sup _{n \in\{0, \ldots, N-1\}} \mathfrak{H}^{n+1}+\sum_{n=0}^{N-1} \Delta t \sum_{\substack{\sigma \in \mathscr{E}_{i n t} \\ \sigma=K \mid L}} \tau_{\sigma}\left[\left(f_{K}^{n+1}-f_{L}^{n+1}\right)^{2}+\left(g_{K}^{n+1}-g_{L}^{n+1}\right)^{2}\right] \leq C .
$$


Proof. We multiply (6) (resp. (7)) by $\Delta t \frac{\log f_{K}^{n+1}}{\mu}$ (resp. $\Delta t \log g_{K}^{n+1}$ ) and sum over $K \in \mathscr{T}$, provides that: $A+B+C=0$, where

$$
\begin{aligned}
A & =\sum_{K \in \mathscr{T}} m_{K}\left[\frac{1}{\mu}\left(f_{K}^{n+1}-f_{K}^{n}\right) \log f_{K}^{n+1}+\left(g_{K}^{n+1}-g_{K}^{n}\right) \log g_{K}^{n+1}\right], \\
B & =\Delta t \sum_{K \in \mathscr{T}} \sum_{\sigma \in \mathscr{E}_{\text {int }, K}} \tau_{\sigma} f_{\sigma}^{n+1}\left(u_{K}^{n+1}-u_{L}^{n+1}\right) \log f_{K}^{n+1}, \\
C & \left.=\Delta t \sum_{K \in \mathscr{T}} \sum_{\sigma \in \mathscr{O}_{\text {int }, K}} \tau_{\sigma} g_{\sigma}^{n+1}\left(v_{K}^{n+1}-v_{L}^{n+1}\right)\right) \log g_{K}^{n+1} .
\end{aligned}
$$

By the convexity of $\Gamma$, we find that

$$
\mathfrak{H}^{n+1}-\mathfrak{H}^{n}=\sum_{K \in \mathscr{T}} m(K)\left[\frac{1}{\mu}\left(\Gamma\left(f_{K}^{n+1}\right)-\Gamma\left(f_{K}^{n}\right)\right)+\Gamma\left(g_{K}^{n+1}\right)-\Gamma\left(g_{K}^{n}\right)\right] \leq A .
$$

We can rewrite $B$ and $C$ as:

$$
\begin{aligned}
& B=\Delta t \sum_{\substack{\sigma \in \mathscr{e}_{\text {int }} \\
\sigma=K \mid L}} \tau_{\sigma} f_{\sigma}^{n+1}\left(u_{K}^{n+1}-u_{L}^{n+1}\right)\left(\log f_{K}^{n+1}-\log f_{L}^{n+1}\right), \\
& C=\Delta t \sum_{\substack{\sigma \in \mathscr{G}_{\text {int }} \\
\sigma=K \mid L}} \tau_{\sigma} g_{\sigma}^{n+1}\left(v_{K}^{n+1}-v_{L}^{n+1}\right)\left(\log g_{K}^{n+1}-\log g_{L}^{n+1}\right) .
\end{aligned}
$$

It follows from the convexity of exp that

$$
a(\log a-\log b) \geq a-b \geq b(\log a-\log b) \quad \forall a, b \in[0,+\infty[,
$$

where we have used the convention $\log (0)=-\infty$ and $0 \log (0)=0$. Hence, in view of the definition (8), one has

$$
\begin{array}{r}
B \geq \Delta t \sum_{\substack{\sigma \in \mathscr{E}_{\text {int }} \\
\sigma=K \mid L}} \tau_{\sigma}\left(\left(f_{K}^{n+1}-f_{L}^{n+1}\right)^{2}+\left(g_{K}^{n+1}-g_{L}^{n+1}\right)\left(f_{K}^{n+1}-f_{L}^{n+1}\right)\right. \\
\left.+\left(b_{K}-b_{L}\right)\left(f_{K}^{n+1}-f_{K}^{n+1}\right)\right), \\
C \geq \Delta t \sum_{\substack{\sigma \in \mathscr{E}_{\text {int }} \\
\sigma=K \mid L}} \tau_{\sigma}\left(\left(g_{K}^{n+1}-g_{L}^{n+1}\right)^{2}+\mu\left(g_{K}^{n+1}-g_{L}^{n+1}\right)\left(f_{K}^{n+1}-f_{L}^{n+1}\right)\right. \\
\left.+\left(b_{K}-b_{L}\right)\left(g_{K}^{n+1}-g_{L}^{n+1}\right)\right) .
\end{array}
$$

Combining these inequalities, one deduces that 


$$
\begin{aligned}
\mathfrak{H}^{n+1}-\mathfrak{H}^{n}+\Delta t \sum_{\substack{\sigma \in \mathscr{E}_{\mathrm{int}} \\
\sigma=K \mid L}} \tau_{\sigma}\left(f_{K}^{n+1}-f_{L}^{n+1}\right)^{2}+\Delta t \sum_{\substack{\sigma \in \mathscr{E}_{\mathrm{int}} \\
\sigma=K \mid L}} \tau_{\sigma}\left(g_{K}^{n+1}-g_{L}^{n+1}\right)^{2} \\
+(\mu+1) \Delta t \sum_{\substack{\sigma \in \mathscr{S}_{\mathrm{int}} \\
\sigma=K \mid L}} \tau_{\sigma}\left(f_{K}^{n+1}-f_{L}^{n+1}\right)\left(g_{K}^{n+1}-g_{L}^{n+1}\right) \\
\leq-\Delta t \sum_{\substack{\sigma \in \mathscr{E}_{\text {int }} \\
\sigma=K \mid L}} \tau_{\sigma}\left(b_{K}-b_{L}\right)\left[\left(f_{K}^{n+1}-f_{L}^{n+1}\right)+\left(g_{K}^{n+1}-g_{L}^{n+1}\right)\right]:=D .
\end{aligned}
$$

Using the Young inequality, one has for all $\varepsilon>0$

$$
D \leq \frac{1}{2 \varepsilon} \Delta t \sum_{\substack{\sigma \in \mathscr{E}_{\mathrm{int}} \\ \sigma=K \mid L}} \tau_{\sigma}\left(b_{K}-b_{L}\right)^{2}+\frac{\varepsilon}{2} \Delta t \sum_{\substack{\sigma \in \mathscr{E}_{\mathrm{int}} \\ \sigma=K \mid L}} \tau_{\sigma}\left[\left(f_{K}^{n+1}-f_{L}^{n+1}\right)+\left(g_{K}^{n+1}-g_{L}^{n+1}\right)\right]^{2} .
$$

We choose $\varepsilon=1+\mu$, we have

$$
\begin{array}{r}
|D| \leq \Delta t \sum_{\substack{\sigma \in \mathscr{E}_{\text {int }} \\
\sigma=K \mid L}} \tau_{\sigma}\left(\frac{1}{2(\mu+1)}\left(b_{K}-b_{L}\right)^{2}+(\mu+1)\left(f_{K}^{n+1}-f_{L}^{n+1}\right)\left(g_{K}^{n+1}-g_{L}^{n+1}\right)\right) \\
+\frac{\mu+1}{2} \Delta t \sum_{\substack{\sigma \in \mathscr{E}_{\text {int }} \\
\sigma=K \mid L}} \tau_{\sigma}\left(\left(f_{K}^{n+1}-f_{L}^{n+1}\right)^{2}+\left(g_{K}^{n+1}-g_{L}^{n+1}\right)^{2}\right) .
\end{array}
$$

Finally, one has

$$
\begin{aligned}
\mathfrak{H}^{n+1}-\mathfrak{H}^{n}+\frac{1-\mu}{2} \Delta t \sum_{\substack{\sigma \in \mathscr{E}_{\text {int }} \\
\sigma=K \mid L}} \tau_{\sigma}\left(f_{K}^{n+1}-f_{L}^{n+1}\right)^{2} \\
+\frac{1-\mu}{2} \Delta t \sum_{\substack{\sigma \in \mathscr{E}_{\text {int }} \\
\sigma=K \mid L}} \tau_{\sigma}\left(g_{K}^{n+1}-g_{L}^{n+1}\right)^{2} \leq \frac{1}{2(\mu+1)} \Delta t \sum_{\substack{\sigma \in \mathscr{E}_{\text {int }} \\
\sigma=K \mid L}} \tau_{\sigma}\left(b_{K}-b_{L}\right)^{2} .
\end{aligned}
$$

Summing over $n=0, \ldots, N-1$, concludes the proof of Proposition 2 .

Proposition 3. For $n \in\{0, \cdots, N-1\}$

$$
\sup _{n \in\{0, \ldots, N-1\}} \mathfrak{E}^{n+1} \leq \mathfrak{E}^{0}
$$

Proof. We multiply (6) (resp. (7)) by $\Delta t \mu u_{K}^{n+1}$ (resp. $\Delta t v_{K}^{n+1}$ ) and sum over $K \in \mathscr{T}$. Summing both equalities and reorganizing the sums, we get $A+B=0$, where 


$$
\begin{gathered}
A=\sum_{K \in \mathscr{T}} m(K)\left[\mu\left(\left(f_{K}^{n+1}+g_{K}^{n+1}+b_{K}\right)-\left(f_{K}^{n}+g_{K}^{n}+b_{K}\right)\right)\left(f_{K}^{n+1}+g_{K}^{n+1}+b_{K}\right)\right] \\
+\sum_{K \in \mathscr{T}} m(K)\left[(1-\mu)\left(\left(g_{K}^{n+1}+b_{K}\right)-\left(g_{K}^{n}+b_{K}\right)\right)\left(g_{K}^{n+1}+b_{K}\right)\right], \\
B=\Delta t \sum_{\substack{\sigma \in \mathscr{E}_{\text {int }} \\
\sigma=K \mid L}} \tau_{\sigma}\left(\mu^{2} f_{\sigma}^{n+1}\left(u_{K}^{n+1}-u_{L}^{n+1}\right)^{2}+g_{\sigma}^{n+1}\left(v_{K}^{n+1}-v_{L}^{n+1}\right)^{2}\right) .
\end{gathered}
$$

We use the following inequality: $(a-b) a \geq \frac{1}{2}\left(a^{2}-b^{2}\right), \forall a, b \in \mathbb{R}$, to get

$$
\begin{aligned}
A \geq \sum_{K \in \mathscr{T}} m(K) & {\left[\frac{\mu}{2}\left(\left(f_{K}^{n+1}+g_{K}^{n+1}+b_{K}\right)^{2}-\left(f_{K}^{n}+g_{K}^{n}+b_{K}\right)^{2}\right)\right] } \\
+ & \sum_{K \in \mathscr{T}} m(K)\left[\frac{1-\mu}{2}\left(\left(g_{K}^{n+1}+b_{K}\right)^{2}-\left(g_{K}^{n}+b_{K}\right)^{2}\right)\right]=\mathfrak{E}^{n+1}-\mathfrak{E}^{n} .
\end{aligned}
$$

Summing over $n=0, \ldots, N-1$, concludes the proof of Proposition 3.

Let us remark that Proposition 2 and Proposition 3 give a discrete $L^{2}\left(0, T ; H^{1}(\Omega)\right)$ and $L^{\infty}\left(0, T ; L^{2}(\Omega)\right)$ bounds on the approximate solutions. These estimates are sufficient to prove the convergence of the scheme when the discretization parameters $\delta$ and $\Delta t$ tend to 0 . We refer to [1] for the proof. As a byproduct, this convergence result ensures the existence of a weak solution to the continuous model. The question of uniqueness of the solution is open as far as we know.

\section{Numerical results}

Let us provide some illustrations of the behaviour of the numerical scheme (6)(8). The scheme leads to a nonlinear system that we solve thanks to the NewtonRaphson method. The numerical analysis of the scheme was carried out for a uniform time discretization of $(0, T)$ only in order to avoid heavy notations. In order to increase the robustness of the algorithm and to ensure the convergence of the Newton-Raphson iterative procedure, we used an adaptive time step procedure in the practical implementation. More precisely, we associate a maximal time step $\Delta t_{\max }$ for the mesh. If the Newton-Raphson method fails to converge after 30 iterations - we choose that the $\ell^{\infty}$ norm of the residual has to be smaller than $10^{-10}$ as stopping criterion-, the time step is divided by two. If the Newton-Raphson method converges, the time step is multiplied by two and projected on $\left[0, \Delta t_{\max }\right]$.

In our test case, the domain is the unit square, i.e., $\Omega=(0,1)^{2}$. We consider an admissible triangular mesh made of 14336 triangles. We choose $b(x, y)=$ $\max \left(0, \frac{1}{2}\left(1-16(x-1 / 2)^{2}\right)((\cos (\pi y)+2))\right.$. We set $\mu=0.9$, and 
$f_{0}(x, y)=\left\{\begin{array}{ll}\frac{1}{2} & \text { if } x \leq \frac{1}{4}, \\ 0 & \text { elsewher, }\end{array} \quad g_{0}(x, y)= \begin{cases}b\left(\frac{1}{2}, 0\right)-b(x, y)-\left(x-\frac{1}{2}\right) & \text { if } x \leq \frac{1}{2} \\ 0 & \text { elsewhere }\end{cases}\right.$

Fig. 2 shows the evolution of $b(x)$ (black), $b(x)+g(x, t)$ (red) and $b(x)+g(x, t)+$ $f(x, t)$ (blue) at different times, and also the evolution of the energy along time. About the model, we observe that there is convergence towards an equilibrium state, with horizontal interfaces as expected.
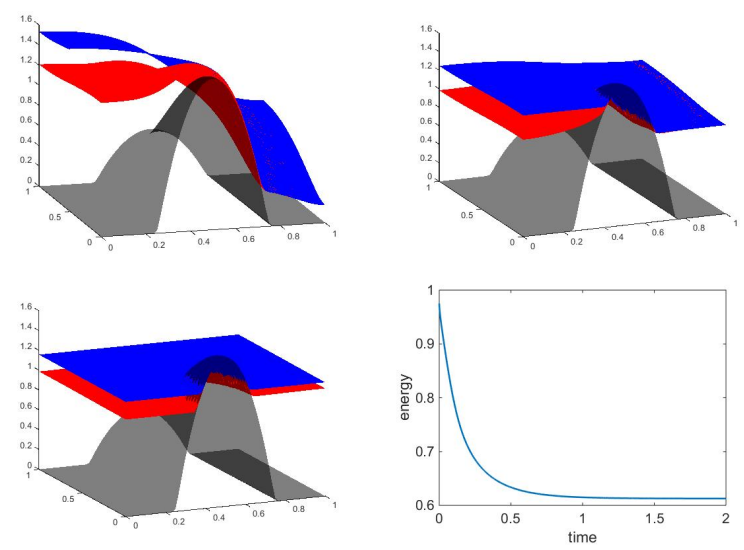

Fig. 2 Behaviour of the model at $t=0.2, t=0.79, t=12$, and evolution of the energy along time

Acknowledgements The author thanks the team Inria/Rapsodi, the Labex CEMPI (ANR-11LABX-0007-01) and the project GEOPOR (ANR-13-JS01-0007-01) for their support.

\section{References}

1. Ait Hammou Oulhaj, A.: Numerical analysis of a finite volume scheme for a seawater intrusion model with cross-diffusion in an unconfined aquifer (2017). HAL: hal-01432197, submitted

2. Escher, J., Laurencot, P., Matioc, B.V.: Existence and stability of weak solutions for a degenerate parabolic system modelling two-phase flows in porous media. In: Annales de l'Institut Henri Poincare (C) Non Linear Analysis, vol. 28, pp. 583-598. Elsevier (2011)

3. Eymard, R., Gallouët, T., Herbin, R.: Finite volume methods. In: Handbook of numerical analysis, Vol. VII, Handb. Numer. Anal., VII, pp. 713-1020. North-Holland, Amsterdam (2000)

4. Jazar, M., Monneau, R.: Derivation of seawater intrusion models by formal asymptotics. SIAM J. Appl. Math. 74(4), 1152-1173 (2014)

5. Jüngel, A.: The boundedness-by-entropy method for cross-diffusion systems. Nonlinearity 28(6), 1963-2001 (2015)

6. Laurençot, P., Matioc, B.V.: A thin film approximation of the Muskat problem with gravity and capillary forces. Journal of the Mathematical Society of Japan 66(4), 1043-1071 (2014) 